\title{
PAIR CORRELATIONS OF QUANTUM CHAOTIC MAPS FROM SUPERSYMMETRY
}

\author{
M R Zirnbauer*
}

November 15, 1997

\begin{abstract}
A conjecture due to Bohigas, Giannoni and Schmit (BGS), stating that the energy level correlations of quantum chaotic systems generically obey the laws of random matrix theory, is given a precise formulation for quantized symplectic maps. No statement is made about any individual quantum map. Rather, a few-parameter ensemble of maps is considered, such that the deterministic map is composed with a diffusion operator on average. The ensemble is a "quantum" one, which is to say that the diffusion operator contracts to the identity in the classical limit. It is argued that the BGS conjecture is true on average over such an ensemble, provided that the classical map is mixing. The method used is closely related to the supersymmetric formalism of Andreev et al for chaotic Hamiltonian systems.
\end{abstract}

\section{Introduction}

One of the goals of semiclassical analysis is to characterize the energy level correlations of quantum mechanical systems in the limit $\hbar \rightarrow 0$. To gain an understanding of these correlations, physicists have traditionally used semiclassical trace formulas, relating the density of energy levels to the periodic orbits of the classical dynamics (see e.g. the contributions by Bogomolny,

\footnotetext{
*Inst. f. Theor. Physik, Universität Köln, Germany; email: zirn@thp.Uni-Koeln.DE
} 
Keating, and Smilansky to this volume). In brief, the qualitative picture that has emerged is that integrable systems have Poisson statistics, whereas for a generic chaotic system one expects random matrix (or Wigner-Dyson) statistics. This picture is supported by a large body of numerical evidence, and applies equally to Hamiltonian systems and symplectic maps. Bohigas, Giannoni, and Schmit (BGS) [1] are credited for having put forth the random matrix conjecture for quantum chaotic systems. The principal development toward an analytical theory is due to Berry [2] who augmented Gutzwiller's trace formula with sum rule arguments to determine the form factor for times much shorter and much longer than the Heisenberg time. Berry's work has recently been refined by Bogomolny and Keating [3] .

In these lectures, I will describe a supersymmetric formalism that offers an alternative to the trace formula approach and promises rigorous results for chaotic symplectic maps. The formalism bears much similarity to that of Andreev et al [4] for chaotic Hamiltonian systems, reviewed at this workshop by Ben Simons. Only the simplest case of maps without unitary or anti-unitary symmetries will be treated here. In particular, time reversal invariance will be assumed to be broken. The starting point of our theory is an expression for the two-level correlation function which involves two ratios of quantum spectral determinants (Sect. 3). The determinants are written as traces over a Fock space of fermions, and the inverse determinants as traces over a Fock space of bosons (Sect. (4). By the introduction of generalized coherent states, the two-level correlation function can then be expressed as a Berezin integral over the Riemannian symmetric superspace of type AIII $\mid A$ III (Sect. 6). A quick review of the basic mathematics underlying the Berezin integral is given in Sect. 5 .

The BGS conjecture is not a precise statement, as the meaning of the word "generic" is left undefined. (The qualification "generic" serves to exclude some prominent counterexamples, such as arithmetic billiards and the cat maps, which are paradigms of classical chaos but fail to obey random matrix statistics when quantized in the canonical way.) It turns out that in our formalism it is impossible to prove the conjecture in the sense originally intended, i.e. as a statement about an individual quantum system. The technical obstacle that prevents us from making progress for individual systems is the nonexistence of the semiclassical limit (Sect. 7) for the Berezin integral representation of the two-level correlation function, or any other correlation function. To enforce such a limit one needs to impose some sort of coarse graining, or ultraviolet regulator, on the Berezin integral. The way to im- 
plement regularization is to compose the map with a stochastic Hamiltonian flow and average (Sect. 8).

From experience with the supersymmetry formalism applied to disordered electron systems [5] it is known that the so-called zero mode approximation to the Berezin integral gives the random matrix answer. The strategy therefore is to establish sufficient conditions for this approximation, which is of the saddle point type, to be justified. The key issue now becomes the stability of the manifold of saddle points. Without ensemble averaging, the Hessian of the saddle point manifold is not positive, and the saddle point approximation is false. Averaging over the stochastic Hamiltonian flow with "time" parameter $\epsilon$ stabilizes the saddle point manifold and leads to a good asymptotic expansion (Sect. 9). If the classical map is mixing we argue from power counting that stability can achieved with a time parameter $\epsilon$ that vanishes with $\hbar$ as $\hbar^{\alpha}$ where $0<\alpha<1$. Thus we propose that the BGS conjecture holds on average over an ensemble of quantum chaotic maps all of which have the same classical limit.

My original intention was to finish with a discussion of the finite $\hbar$ corrections to the universal random matrix result. These are a very timely issue, as the ballistic nonlinear $\sigma$ model calculation of Andreev et al disagrees with the result of Bogomolny and Keating derived from the diagonal approximation. (The difference lies in the weights given to the contributions from periodic orbits with repetition number $r \geq 2$.) There exist strong indications that the result of Bogomolny and Keating is the correct one. I believe that the resolution of the discrepancy will teach us something profound about the ballistic nonlinear $\sigma$ model, but this definitely exceeds the scope of the present lectures and must be left for a future publication.

\section{Preliminaries}

We begin with some basic definitions.

Let $M$, called the phase space, be a $2 d$-dimensional compact manifold with a symplectic structure $\omega$, i.e. an antisymmetric second rank tensor that is expressed in local coordinates $q_{k}, p_{k}$ by $\omega=\sum_{k=1}^{d} \mathrm{~d} p_{k} \wedge \mathrm{d} q_{k}$. Via $\omega$, every function $f: M \rightarrow \mathbb{R}$ is associated with a Hamiltonian vector field $\Xi_{f}=\sum_{k=1}^{d}\left(\frac{\partial f}{\partial p_{k}} \frac{\partial}{\partial q_{k}}-\frac{\partial f}{\partial q_{k}} \frac{\partial}{\partial p_{k}}\right)$. The volume element, or Liouville measure, on $M$ is denoted by $d x:=\omega^{\wedge d}$. A map $\chi: M \rightarrow M$ is called symplectic (or area preserving for $d=1$ ) if it preserves $\omega$ and, consequently, the Liouville 
measure. The map $\chi$ acts on functions $f: M \rightarrow \mathbb{R}$ by $\left(\chi^{*} f\right)(x)=f(\chi(x))$. The dual of its inverse, $\chi^{-1^{*}}$, is called the Frobenius-Perron operator. A little computation,

$$
\begin{aligned}
& \left(\chi^{-1^{*}} f\right)(x)=f\left(\chi^{-1}(x)\right) \\
= & \int_{M} d y \delta\left(\chi^{-1}(x)-y\right) f(y)=\int_{M} d y \delta(x-\chi(y)) f(y),
\end{aligned}
$$

shows that the Frobenius-Perron operator has the integral kernel

$$
\left(\chi^{-1^{*}}\right)(x, y)=\delta(x-\chi(y))
$$

which identifies $\chi^{-1 *}$ as the propagator (for one time step) of the classical dynamics obtained by iterating $\chi$.

Quantization turns every smooth function $f: M \rightarrow \mathbb{R}$ into a self-adjoint operator $\mathcal{Q}_{N}(f)=F_{N}$ on a Hilbert space $\mathcal{H}_{N}$ of finite dimension $N=$ $(2 \pi \hbar)^{-d} \int_{M} d x$. The quantization of a smooth phase space function is called a pseudodifferential operator. The quantum analog of the classical map $\chi$ is a unitary operator $U_{N}: \mathcal{H}_{N} \rightarrow \mathcal{H}_{N}$, whose eigenvalues we denote by $e^{i \theta_{n}}(n=1, \ldots, N)$. The $\theta_{n}$ 's are sometimes called quasienergies. Let $\mathcal{D}_{N}=\mathcal{H}_{N} \otimes \mathcal{H}_{N}^{*} \simeq \operatorname{End}\left(\mathcal{H}_{N}\right)$ be the space of linear operators on $\mathcal{H}_{N}$. The unitary operator $U_{N}$ acts on $\mathcal{D}_{N}$ by conjugation. More precisely, the quantum time evolution (by one time step) of an element $F_{N} \in \mathcal{D}_{N}$ is given by

$$
\operatorname{Ad}\left(U_{N}\right)^{-1} F_{N}=U_{N}^{-1} F_{N} U_{N}
$$

(The notation $\operatorname{Ad}(U)$ for conjugation by $U$ is taken from Lie algebra theory.) In the classical limit $\hbar \rightarrow 0, \operatorname{Ad}\left(U_{N}\right)^{-1}$ acting on a pseudodifferential operator $F_{N}=\mathcal{Q}_{N}(f)$ approaches the Frobenius-Perron operator:

$$
\operatorname{Ad}\left(U_{N}\right)^{-1} F_{N} \stackrel{\hbar \rightarrow 0}{\longrightarrow} \mathcal{Q}_{N}\left(\chi^{-1^{*}} f\right)
$$

Examples illustrating these general definitions are furnished by the cat maps [6]. The phase space in this case is the two-torus $\mathrm{T}^{2}$, coordinatized by a pair of canonically conjugate position and momentum functions $q$ and $p$, which are defined modulo 1 . For a concrete example, consider the cat map $\chi: \mathrm{T}^{2} \rightarrow \mathrm{T}^{2}$ that acts on $q$ and $p$ as

$$
\begin{aligned}
& q \circ \chi=q+2 p \quad(\bmod 1), \\
& p \circ \chi=2 q+5 p \quad(\bmod 1)
\end{aligned}
$$


which entails a Frobenius-Perron operator expressed by

$$
\chi^{-1^{*}}=\exp (-2 q \partial / \partial p) \exp (-2 p \partial / \partial q)
$$

on differentiable functions. [Note that $\exp (-\partial / \partial q)=\exp (-\partial / \partial p)=1$, so that $\exp (-2 p \partial / \partial q)$ and $\exp (-2 q \partial / \partial p)$ are globally well-defined, although $p \partial / \partial q$ and $q \partial / \partial p$ are not.] The locally defined vector fields $\Xi_{f}=2 q \partial / \partial p$ and $\Xi_{g}=2 p \partial / \partial q$ derive from locally defined functions $f=-q^{2}$ and $g=p^{2}$, and quantization of $\chi$ yields a unitary operator

$$
U_{N}=\exp \left(i P_{N}^{2} / \hbar\right) \exp \left(-i Q_{N}^{2} / \hbar\right)
$$

where $\hbar^{-1}=2 \pi N \int_{0}^{1} \mathrm{~d} p \int_{0}^{1} \mathrm{~d} q=2 \pi N$. In a basis where $\exp \left(2 \pi i P_{N}\right)$ is diagonal with the eigenvalues being the $N^{\text {th }}$ roots of unity $\exp (2 \pi i n / N)(n=$ $1,2, \ldots, N), U_{N}$ has the following matrix

$$
\left(U_{N}\right)_{n n^{\prime}}=\frac{1}{\sqrt{N}} \exp \frac{2 \pi i}{N}\left(n^{2}+\left(n-n^{\prime}\right)^{2}\right) .
$$

\section{BGS conjecture formulated precisely}

There exist many statistical measures of level correlations. The one we will focus on here is the so-called pair correlation function, defined on a test function $f: \mathrm{U}(1) \rightarrow \mathbb{C}$ by

$$
C(f)=\frac{2 \pi}{N^{2}} \sum_{m, n=1}^{N} f\left(e^{i\left(\theta_{m}-\theta_{n}\right)}\right)-\int_{0}^{2 \pi} f\left(e^{i \theta}\right) \mathrm{d} \theta .
$$

If we take the liberty of choosing for $f$ the Dirac $\delta$-distribution centered at $e^{i \varphi}, \delta_{\varphi}\left(e^{i \theta}\right)=(2 \pi)^{-1} \sum_{l \in \mathbb{Z}} e^{i l(\theta-\varphi)}$, we get the two-level correlation function

$$
R_{2}(\theta)=C\left(\delta_{\theta}\right)=\frac{2}{N^{2}} \sum_{l=1}^{\infty} \cos (l \theta)\left|\operatorname{Tr} U^{l}\right|^{2} .
$$

For our purposes, it is technically convenient to consider in addition to $R_{2}$ the following correlator of determinants:

$$
\Omega\left(\gamma_{+0}, \gamma_{+1} ; \gamma_{-0}, \gamma_{-1}\right)=\int_{0}^{2 \pi} \frac{\mathrm{d} \theta}{2 \pi} \frac{\operatorname{Det}\left(1-\gamma_{+1} e^{i \theta} U_{N}\right) \operatorname{Det}\left(1-\gamma_{-1} e^{-i \theta} U_{N}^{-1}\right)}{\operatorname{Det}\left(1-\gamma_{+0} e^{i \theta} U_{N}\right) \operatorname{Det}\left(1-\gamma_{-0} e^{-i \theta} U_{N}^{-1}\right)}
$$


where $\gamma_{ \pm 0}, \gamma_{ \pm 1}$ are complex parameters. Note that the two-level correlation function can be extracted from $\Omega$ by taking two derivatives:

$$
R_{2}(\theta)=-\left.\frac{1}{2 N^{2}} \frac{\partial^{2}}{\partial \varphi \partial \varphi^{\prime}} \operatorname{Re} \Omega\left(e^{i \theta+i \varphi}, e^{i \theta-i \varphi} ; e^{i \varphi^{\prime}}, e^{-i \varphi^{\prime}}\right)\right|_{\varphi=\varphi^{\prime}=0} .
$$

In Sects. 46 I will sketch the derivation of a formula for $\Omega$ that underlies the supersymmetric method for quantized maps, and I will go over some of the basic mathematics needed. Before doing so, it is proper to motivate the reader by giving an outline of the results that can be obtained.

The first and very important message is that our method gives no answer at all for the pair correlation function, or any other correlation function, of an individual quantized map. (This is to be contrasted with what has been claimed by Andreev et al.) For reasons that will be explained later on, we can make a statement only about the expected value of the correlations for an ensemble of quantized maps over a well-chosen probability space.

Let me therefore digress and describe this ensemble. We pick a finite number $s$ of Hamiltonian functions $x_{k}: M \rightarrow \mathbb{R}$, with associated Hamiltonian vector fields $\Xi_{k}$, and Hamiltonian operators $X_{k, N}: \mathcal{H}_{N} \rightarrow \mathcal{H}_{N}$. The latter we multiply by uncorrelated Gaussian random variables $\xi_{k}$ with zero mean and $\hbar$-dependent variances $\left\langle\xi_{k}^{2}\right\rangle=2 \epsilon(\hbar)$, and we consider instead of a single $U_{N}$ an $s$-parameter family of unitary operators

$$
U_{N}(\xi):=\exp \left(i \sum_{k=1}^{s} \xi_{k} X_{k, N} / \hbar\right) U_{N}
$$

The choice of Hamiltonians $x_{k}$ is constrained by the requirement that the weighted sum of squares $\sum_{k=1}^{s} \Xi_{k}^{2}$ be an elliptic operator.

The good news is that we can get an answer for the expected correlation functions $\langle\Omega\rangle$ and $\left\langle R_{2}\right\rangle$ by taking $\epsilon(\hbar)=$ const $\times \hbar^{\alpha}$ where $\alpha$ is a number between 0 and 1 (and we may choose $\alpha=1 / 2$, say). This dependence of the variances on $\hbar$ means that, roughly speaking, all members of the ensemble have the same classical limit. In other words, the ensemble we use is "quantum", and we could say that what we are averaging over are different quantizations of the same classical map.

Now, let the classical map be mixing, and arrange for the quantized map to have no unitary or anti-unitary symmetries. We will argue that the expected correlation functions in this case have the following semiclassical limits:

$$
\lim _{N \rightarrow \infty}\left\langle\Omega\left(e^{i c_{+0} / N}, e^{i c_{+1} / N} ; e^{-i c_{-0} / N}, e^{-i c_{-1} / N}\right)\right\rangle=
$$




$$
1-\frac{\left(c_{+0}-c_{+1}\right)\left(c_{-0}-c_{-1}\right)}{\left(c_{+0}-c_{-0}\right)\left(c_{+1}-c_{-1}\right)}\left(1-e^{i\left(c_{+1}-c_{-1}\right)}\right)
$$

and

$$
\lim _{N \rightarrow \infty}\left\langle R_{2}(2 \pi x / N)\right\rangle=\delta(x)-\sin ^{2}(\pi x) /(\pi x)^{2} .
$$

To put this result into context, we observe that our expressions for $\langle\Omega\rangle$ and $\left\langle R_{2}\right\rangle$ coincide with those for a random unitary matrix in the large $-N$ limit. What we propose, then, is a precise formulation of the BGS conjecture: random matrix theory applies to chaotic maps on average over a quantum ensemble of the kind specified. More specifically, although there exist counterexamples such as the cat maps, these are a set of measure zero in parameter space, and the random matrix result does obtain on averaging.

\section{Supersymmetric formula for maps (I)}

In what follows we shall construct a novel and useful integral representation of the correlator $\Omega$. Our first step will be to express the determinants $\operatorname{Det}\left(1-\gamma U_{N}\right)^{ \pm 1}$ as traces over Fock space. To that end, let $\mathcal{F}_{N}$ be the $2^{N_{-}}$ dimensional fermionic Fock space that is generated by acting with creation operators $f_{n}^{\dagger}(n=1,2, \ldots, N)$ on a particle vacuum $|0\rangle$. The operators $f_{n}^{\dagger}$ and their adjoints $f_{n}$ satisfy the canonical anticommutation relation for fermions: $f_{n}^{\dagger} f_{n^{\prime}}+f_{n^{\prime}} f_{n}^{\dagger}=\delta_{n n^{\prime}}$.

Now we claim that determinants such as those appearing in the numerator of the defining expression for $\Omega$ can be written as Fock space traces:

$$
\operatorname{Det}\left(1-U_{N}\right)=\operatorname{Tr}_{\mathcal{F}_{N}}(-1)^{N_{F}} \exp \sum_{n, n^{\prime}=1}^{N} f_{n}^{\dagger}\left(\ln U_{N}\right)_{n n^{\prime}} f_{n^{\prime}}
$$

where $N_{F}=\sum_{n} f_{n}^{\dagger} f_{n}$ is the fermion number. To prove that claim, we may assume the matrix $U_{N}$ to be diagonal as both sides of the equation are invariant under a change of the single particle basis. Put $\left(U_{N}\right)_{n n^{\prime}}=\lambda_{n} \delta_{n n^{\prime}}$. Then the above equation is verified by the following simple computation:

$$
\begin{aligned}
\prod_{n=1}^{N}\left(1-\lambda_{n}\right) & =\prod_{n=1}^{N}\left(e^{0 \times \ln \lambda_{n}}-e^{1 \times \ln \lambda_{n}}\right) \\
& =\prod_{n=1}^{N} \operatorname{Tr}_{\mathcal{F}_{1}}(-1)^{f_{1}^{\dagger} f_{1}} \exp \left(f_{1}^{\dagger} f_{1} \ln \lambda_{n}\right)
\end{aligned}
$$




$$
=\operatorname{Tr}_{\mathcal{F}_{N}}(-1)^{N_{F}} \exp \sum_{n=1}^{N} f_{n}^{\dagger} f_{n} \ln \lambda_{n} .
$$

The multivaluedness of the logarithm does not cause any ambiguity here as $\exp 2 \pi i f_{n}^{\dagger} f_{n}=1$.

A similar expression for the inverse of a determinant can be obtained by substituting bosons for fermions. Let $\mathcal{B}_{N}$ be the bosonic Fock space generated by canonical boson operators $b_{n}^{\dagger}, b_{n}$ with commutation relations $b_{n^{\prime}} b_{n}^{\dagger}-b_{n}^{\dagger} b_{n^{\prime}}=\delta_{n n^{\prime}}$. For $|\gamma|<1$ we have the identity

$$
\operatorname{Det}\left(1-\gamma U_{N}\right)^{-1}=\operatorname{Tr}_{\mathcal{B}_{N}} \exp \sum_{n, n^{\prime}=1}^{N} b_{n}^{\dagger}\left(\ln \gamma U_{N}\right)_{n n^{\prime}} b_{n^{\prime}} .
$$

Again, this is verified by diagonalizing $U_{N}$ and computing a single boson trace,

$$
\operatorname{Tr}_{\mathcal{B}_{1}} e^{b_{1}^{\dagger} b_{1} \ln \lambda}=e^{0 \times \ln \lambda}+e^{1 \times \ln \lambda}+\cdots+e^{n \ln \lambda}+\cdots=(1-\lambda)^{-1} .
$$

The inequality $|\gamma|<1$ is needed in order for the sum on the right hand side to converge.

Let us now assemble the various pieces to build a formula for $\Omega$. There are two determinants in both the numerator and the denominator, so we introduce two fermionic and two bosonic Fock spaces $\mathcal{F}_{N}^{ \pm}$and $\mathcal{B}_{N}^{ \pm}$, and write

$$
\begin{aligned}
\frac{\operatorname{Det}\left(1-\gamma_{+1} U_{N}\right) \operatorname{Det}\left(1-\gamma_{-1} U_{N}^{-1}\right)}{\operatorname{Det}\left(1-\gamma_{+0} U_{N}\right) \operatorname{Det}\left(1-\gamma_{-0} U_{N}^{-1}\right)} \\
=\operatorname{Tr}_{\mathcal{B}_{N}^{+} \otimes \mathcal{F}_{N}^{+} \otimes \mathcal{B}_{N}^{-} \otimes \mathcal{F}_{N}^{-}(-1)^{N_{F}} \exp \sum_{n n^{\prime}}}\left(b_{+n}^{\dagger}\left(\ln \gamma_{+0} U_{N}\right)_{n n^{\prime}} b_{+n^{\prime}}\right. \\
+b_{-n}^{\dagger}\left(\ln \gamma_{-0} U_{N}^{-1}\right)_{n n^{\prime}} b_{-n^{\prime}} \\
+f_{+n}^{\dagger}\left(\ln \gamma_{+1} U_{N}\right)_{n n^{\prime}} f_{+n^{\prime}} \\
\left.+f_{-n}^{\dagger}\left(\ln \gamma_{-1} U_{N}^{-1}\right)_{n n^{\prime}} f_{-n^{\prime}}\right) .
\end{aligned}
$$

Here $N_{F}=\sum_{n=1}^{N}\left(f_{+n}^{\dagger} f_{+n}+f_{-n}^{\dagger} f_{-n}\right)$, and convergence of the sums requires $\left|\gamma_{ \pm 0}\right|<1$. To obtain $\Omega$ we need to replace $U_{N}$ by $e^{i \theta} U_{N}$ and integrate over $\theta$. If $N_{ \pm}=\sum_{n}\left(b_{ \pm n}^{\dagger} b_{ \pm n}+f_{ \pm n}^{\dagger} f_{ \pm n}\right)$ counts the number of retarded $(+)$ or advanced $(-)$ particles, this produces an extra factor

$$
\int_{0}^{2 \pi} \frac{d \theta}{2 \pi}\left(e^{i \theta}\right)^{N_{+}-N_{-}}=: P
$$


under the trace. The operator $P$ gives unity when applied to a state $|\psi\rangle$ with $\left(N_{+}-N_{-}\right)|\psi\rangle=0$, and zero otherwise, and thus projects the super Fock space $\mathcal{B}_{N}^{+} \otimes \mathcal{F}_{N}^{+} \otimes \mathcal{B}_{N}^{-} \otimes \mathcal{F}_{N}^{-}$onto the subspace with equal retarded and advanced particle numbers. Hence, on writing $\sum_{n n^{\prime}} b_{+n}^{\dagger}\left(\ln \gamma_{+0} U_{N}\right)_{n n^{\prime}} b_{+n^{\prime}}=$ : $b_{+}^{\dagger} \ln \left(\gamma_{+0} U_{N}\right) b_{+}$etc. to simplify the notation, we obtain

$$
\begin{aligned}
\Omega\left(\gamma_{+0}, \gamma_{+1} ; \gamma_{-0}, \gamma_{-1}\right)= & \\
\operatorname{Tr}_{N_{+}=N_{-}}(-1)^{N_{F}} \exp \left(b_{+}^{\dagger} \ln \left(\gamma_{+0} U_{N}\right) b_{+}\right. & +b_{-}^{\dagger} \ln \left(\gamma_{-0} U_{N}^{-1}\right) b_{-} \\
& \left.+f_{+}^{\dagger} \ln \left(\gamma_{+1} U_{N}\right) f_{+}+f_{-}^{\dagger} \ln \left(\gamma_{-1} U_{N}^{-1}\right) f_{-}\right) .
\end{aligned}
$$

The final step in the construction of the supersymmetric formula for $\Omega$ is to pass from the trace over super Fock space to a Berezin integral, by the introduction of generalized coherent states. This step is perhaps unfamiliar and deserves a certain amount of explanation.

Let me first illustrate the basic idea at the example of $\gamma_{+0}=\gamma_{-0}=0$, in which case the bosonic degrees of freedom are absent. Thus, we start from the relation

$$
\Omega\left(0, \gamma_{+1} ; 0, \gamma_{-1}\right)=\operatorname{Tr}_{\mathcal{F}_{N}^{+} \otimes \mathcal{F}_{N}^{-}} P_{F} \exp \left(f_{+}^{\dagger} \ln \left(\gamma_{+1} U_{N}\right) f_{+}+f_{-}^{\dagger} \ln \left(\gamma_{-1} U_{N}^{-1}\right) f_{-}\right)
$$

where $P_{F}=(2 \pi)^{-1} \int_{0}^{2 \pi} \mathrm{d} \theta \exp i \theta \sum_{n}\left(f_{+n}^{\dagger} f_{+n}-f_{-n}^{\dagger} f_{-n}\right)$. Consider then the BCS (or pair) coherent states

$$
|Z\rangle=\exp \left(\sum_{n, n^{\prime}=1}^{N} f_{+n}^{\dagger} Z_{n n^{\prime}} f_{-n^{\prime}}^{\dagger}\right)|0\rangle
$$

with complex amplitudes $Z_{n n^{\prime}}$. Being made from pairs of one retarded and one advanced particle, these states satisfy the constraint $P_{F}|Z\rangle=|Z\rangle$. Moreover, they enjoy the key property of providing a resolution of the projector $P_{F}$ :

$$
P_{F}=\int_{\mathbb{C}^{N \times N}} d \mu_{N}(Z, \bar{Z})|Z\rangle\langle Z|,
$$

where $d \mu_{N}(Z, \bar{Z})=$ const $\times \operatorname{Det}\left(1+Z^{\dagger} Z\right)^{-2 N-1} \prod_{n, n^{\prime}=1}^{N} \mathrm{~d} Z_{n n^{\prime}} \mathrm{d} \bar{Z}_{n n^{\prime}}$. The proof proceeds via Schur's lemma, by demonstrating that $\int d \mu_{N}(Z, \bar{Z})|Z\rangle\langle Z|$ commutes with all of the operators $f_{+n}^{\dagger} f_{-n^{\prime}}$ and $f_{-n}^{\dagger} f_{+n^{\prime}}\left(n, n^{\prime}=1, \ldots, N\right)$. A standard computation on coherent states []] yields

$$
\begin{array}{r}
\left\langle Z\left|\exp \sum_{n n^{\prime}}\left(f_{+}^{\dagger} \ln \left(\gamma_{+1} U_{N}\right) f_{+}+f_{-}^{\dagger} \ln \left(\gamma_{-1} U_{N}^{-1}\right) f_{-}\right)\right| Z\right\rangle \\
=\operatorname{Det}\left(1+\gamma_{+1} \gamma_{-1} Z^{\dagger} U_{N} Z U_{N}^{-1}\right) .
\end{array}
$$


Hence, by inserting the coherent state resolution of $P_{F}$ into the expression for $\Omega\left(0, \gamma_{+1} ; 0, \gamma_{-1}\right)$, we arrive at the formula

$$
\Omega\left(0, \gamma_{+1} ; 0, \gamma_{-1}\right)=\int_{\mathbb{C}^{N \times N}} d \mu_{N}(Z, \bar{Z}) \operatorname{Det}\left(1+\gamma_{+1} \gamma_{-1} Z^{\dagger} U_{N} Z U_{N}^{-1}\right)
$$

Our aim now is to extend this formula to allow for a nonvanishing first and third argument of $\Omega$, in which case the Fock space expression involves both fermions and bosons. This requires a supergeneralization of the notion of coherent states and their integration measure. The purpose of the next section is to review some of the basic mathematics needed. We will return to the task of expressing $\Omega$ as a coherent state integral in Sect. 6 .

\section{Basic notions of analysis on supermanifolds}

Familiarity with the elementary theory of differentiable manifolds is assumed. In the present section the summation convention is in force.

Let $\Lambda \mathbb{R}^{q}$ be the Grassmann algebra over $\mathbb{R}$ with $q$ generators $\psi^{1}, \psi^{2}, \ldots, \psi^{q}$. By definition, the Grassmann generators anticommute: $\psi^{i} \psi^{j}+\psi^{j} \psi^{i}=0$. They are known to physicists from their use in the construction of path integrals for fermions. Note in particular $\psi^{i} \psi^{i}=-\psi^{i} \psi^{i}=0$.

Now pick some domain $U \subset \mathbb{R}^{p}$. A map $F$ from $U$ into the Grassmann algebra,

$$
\begin{aligned}
F: U & \rightarrow \Lambda \mathbb{R}^{q} \\
a & \mapsto F_{0}(a)+F_{i}(a) \psi^{i}+F_{i_{1} i_{2}}(a) \psi^{i_{1}} \psi^{i_{2}}+\cdots+F_{i_{1} \cdots i_{q}}(a) \psi^{i_{1}} \cdots \psi^{i_{q}},
\end{aligned}
$$

will be referred to as a superfunction (or simply a function) on $U$. $F_{0}$ is called the number part of $F$. We distinguish between even and odd superfunctions (the former are even and the latter are odd in the Grassmann generators). This distinction endows the algebra of superfunctions with a $\mathbb{Z}_{2}-$ grading.

We can now describe what is meant by a $(p, q)$-dimensional real-analytic supermanifold in the sense of Berezin, Kostant and Leites [8]. Take a (realanalytic) manifold $M$ of dimension $p$ and cover it by domains $U_{1}, U_{2}, \ldots$. Let $p$ even and $q$ odd coordinate superfunctions be given on each domain. [For the purpose of illustration, we denote these by

$$
\begin{array}{ll} 
& x^{1}, \ldots, x^{p} ; \xi^{1}, \ldots, \xi^{q} \quad \text { on } U_{1}, \\
\text { and by } & \left.y^{1}, \ldots, y^{p} ; \eta^{1}, \ldots, \eta^{q} \quad \text { on } U_{2} .\right]
\end{array}
$$


Assume that transition functions exist on overlapping domains and are consistent with the $\mathbb{Z}_{2}$-grading. [For example, on $U_{1} \cap U_{2}$ this means that there are relations

$$
\begin{aligned}
& y^{i}=f^{i}(x, \xi)=f_{0}^{i}(x)+f_{k l}^{i}(x) \xi^{k} \xi^{l}+\cdots \\
& \eta^{j}=\varphi^{j}(x, \xi)=\varphi_{k}^{j}(x) \xi^{k}+\varphi_{k l m}^{j}(x) \xi^{k} \xi^{l} \xi^{m}+\cdots
\end{aligned}
$$

(and the corresponding inverse relations) with $f_{0}^{i}, f_{k l}^{i}, \varphi_{k}^{j}, \varphi_{k l m}^{j}$ etc. being functions on a subset of $\mathbb{R}^{p}$.] If the transition functions are analytic functions, we call the algebra of superfunctions on $M$ generated by the coordinate superfunctions a real-analytic supermanifold of dimension $(p, q)$. Complexanalytic supermanifolds are defined in an analogous manner (just replace $\Lambda \mathbb{R}^{q}$ by $\Lambda \mathbb{C}^{q}$, the Grassmann algebra over $\mathbb{C}$ with $q$ generators, and require the transition functions to be holomorphic). The manifold $M$ is called the base of the supermanifold.

Of course, a proper definition avoids any reference to specific coordinates, and in a more mathematical exposition [9] one would define a supermanifold to be a "sheaf of algebras of superfunctions ...". However, the coordinate description given here is good enough for our purposes.

The standard example of a complex-analytic supermanifold is $\mathbb{C P}^{1 \mid 1}$. Let $z^{1}, z^{2}$ be canonical coordinates of $\mathbb{C}^{2}$, and consider the algebra of superfunctions: $\mathbb{C}^{2} \backslash\{0\} \rightarrow \Lambda \mathbb{C}^{1}$, with the complex Grassmann generator of $\Lambda \mathbb{C}^{1}$ denoted by $\zeta^{1}$. Then focus on the subalgebra of superfunctions which are homogeneous of degree zero in these generators, i.e. are invariant under rescalings $z^{1} \rightarrow c z^{1}, z^{2} \rightarrow c z^{2}, \zeta^{1} \rightarrow c \zeta^{1}$ with $c \in \mathbb{C} \backslash\{0\}$. This algebra is what is called the complex projective superspace $\mathbb{C P}^{1 \mid 1}$. Its base is the ordinary complex projective space $\mathbb{C P}^{1}$. Because the null element has been removed from $\mathbb{C}^{2}$, at least one of the two coordinates $z^{1}, z^{2}$ is nonzero. Let $U_{1}$ be the domain where $z^{1} \neq 0$. Then homogeneity means

$$
f\left(z^{1}, z^{2}, \zeta^{1}\right)=f\left(1, z^{2} / z^{1}, \zeta^{1} / z^{1}\right),
$$

so that $f$ is a function of two variables $z:=z^{2} / z^{1}$ and $\zeta:=\zeta^{1} / z^{1}$, which are taken to be the coordinate (super)functions on $U_{1}$. Similarly on $U_{2}$, defined by $z^{2} \neq 0$, we have

$$
f\left(z^{1}, z^{2}, \zeta^{1}\right)=f\left(z^{1} / z^{2}, 1, \zeta^{1} / z^{2}\right)
$$

and here we put $z^{\prime}:=z^{1} / z^{2}$ and $\zeta^{\prime}:=\zeta^{1} / z^{2}$. The transition functions on 
$U_{1} \cap U_{2}$ are then given by

$$
z^{\prime}=\frac{1}{z}, \quad \zeta^{\prime}=\frac{\zeta}{z} .
$$

The domains $U_{1}$ and $U_{2}$ cover the base $\mathbb{C P}^{1}$, and the transition functions are holomorphic, so $\mathbb{C} \mathrm{P}^{1 \mid 1}$ is a complex-analytic supermanifold, the complex dimension being $(1,1)$.

Of the many structures that exist on supermanifolds, the most important one for our purposes is the Berezin integral. To define it, we must first introduce the notion of Berezin form, also called superintegration measure or integral superform. If $\mathcal{A}$ denotes the algebra of superfunctions of our supermanifold and $\Omega^{*} M$ the space of volume forms on the base $M$, a Berezin form $\omega$ is a linear and local map

$$
\begin{aligned}
\omega: \mathcal{A} & \rightarrow \Omega^{*} M, \\
f & \mapsto \omega[f],
\end{aligned}
$$

i.e. a linear and local rule for converting superfunctions into volume forms on $M$. This process of conversion is sometimes called the "Fermi integral" or "integration over the Grassmann variables".

For example, an interesting class of Berezin forms on $\mathbb{C P}^{1 \mid 1}$ is

$$
\omega^{(n)}=\mathrm{d} z \wedge \mathrm{d} \bar{z} \circ \frac{\partial^{2}}{\partial \zeta \partial \bar{\zeta}} \circ(1+\bar{z} z+\bar{\zeta} \zeta)^{-n}
$$

for $n \in N$. The meaning hereof is this: to compute $\omega^{(n)}[f]$, one first multiplies the superfunction $f$ by $(1+\bar{z} z+\bar{\zeta} \zeta)^{-n}$, then one takes two derivatives $\partial^{2} / \partial \zeta \partial \bar{\zeta}$ (thereby removing the Grassmann generators), and finally one multiplies by $\mathrm{d} z \wedge \mathrm{d} \bar{z}$ to obtain a two-form on $\mathbb{C P}^{1}$. To illustrate, we take

$$
f=f_{0}(z, \bar{z})+f_{01}(z, \bar{z}) \bar{\zeta}+f_{10}(z, \bar{z}) \zeta+f_{11}(z, \bar{z}) \bar{\zeta} \zeta,
$$

in which case we get

$$
\omega^{(n)}[f]=\left(\frac{f_{11}(z, \bar{z})}{(1+\bar{z} z)^{n}}-n \frac{f_{00}(z, \bar{z})}{(1+\bar{z} z)^{n+1}}\right) \mathrm{d} z \wedge \mathrm{d} \bar{z} .
$$

A special role is played by the Berezin form $\omega^{(1)}$. Converting $\omega^{(1)}$ from one chart to another by using the transition functions $z=1 / z^{\prime}$ and $\zeta=\zeta^{\prime} / z^{\prime}$, one 
finds that $\omega^{(1)}$ operates by the same expression in both charts. (Moreover, $\omega^{(1)}$ is invariant under an action of the Lie supergroup $\mathrm{SU}(2 \mid 1)$ on $\mathbb{C} \mathrm{P}^{1 \mid 1}$.)

The definition of Berezin forms immediately leads to a notion of integration on supermanifolds called the Berezin integral, or superintegral. Since a Berezin form $\omega$ converts a superfunction $f$ into a volume form $\omega[f]$ on $M$, the natural thing to do is to integrate $\alpha:=\omega[f]$ in the ordinary fashion to produce the number $\int_{M} \alpha$. Thus a Berezin integral is defined to be a composite map

$$
f \mapsto \omega[f] \mapsto \int_{M} \omega[f]
$$

taking superfunctions into the complex numbers. Note that this definition decrees the Berezin integral to be a two-step process: first the "integration" (or, rather, differentiation) of the Grassmann generators is done, and afterwards the differential form $\omega[f]$ is integrated in the usual sense. The advanced user occasionally finds it convenient to deviate from this rule and perform part of the ordinary integrals first. However, in case of doubt the definition recited [9] above is the one to go back to.

Ordinary integration is coordinate independent, i.e. one has the option of changing integration variables by the substitution rule. It turns out that this option also exists for superintegrals, albeit with a certain caveat. To describe the relevant statement, which is due to Berezin, we introduce the short hand notation

$$
D(x, \xi):=\mathrm{d} x^{1} \wedge \ldots \wedge \mathrm{d} x^{p} \frac{\partial}{\partial \xi^{1}} \cdots \frac{\partial}{\partial \xi^{q}},
$$

and consider a change of variables (consistent with the $\mathbb{Z}_{2}$-grading) from $x, \xi$ to $y, \eta$ by

$$
\begin{array}{rlr}
y^{i}=f^{i}\left(x^{1}, \ldots, x^{p} ; \xi^{1}, \ldots, \xi^{q}\right) & (i=1, \ldots, p), \\
\eta^{j}=\varphi^{j}\left(x^{1}, \ldots, x^{p} ; \xi^{1}, \ldots, \xi^{q}\right) & (j=1, \ldots, q) .
\end{array}
$$

The role of the Jacobian of ordinary analysis is taken by the Berezinian,

$$
\operatorname{Ber}\left(\frac{y, \eta}{x, \xi}\right):=\operatorname{SDet}\left(\begin{array}{cc}
\partial f^{i} / \partial x^{j} & \partial f^{i} / \partial \xi^{j} \\
\partial \varphi^{i} / \partial x^{j} & \partial \varphi^{i} / \partial \xi^{j}
\end{array}\right)
$$

where the superdeterminant SDet of a supermatrix $\left(\begin{array}{cc}A & B \\ C & D\end{array}\right)$ (with the matrix elements of $A, D$ being even and those of $B, C$ odd) is defined by

$$
\operatorname{SDet}\left(\begin{array}{cc}
A & B \\
C & D
\end{array}\right)=\frac{\operatorname{Det}\left(A-B D^{-1} C\right)}{\operatorname{Det} D}=\frac{\operatorname{Det} A}{\operatorname{Det}\left(D-C A^{-1} B\right)} \text {. }
$$


Berezin's theorem [9] states that the substitution rule is valid, i.e.

$$
\int D(y, \eta) f=\int D(x, \xi) \operatorname{Ber}\left(\frac{y, \eta}{x, \xi}\right) f
$$

provided that $f$ is compactly supported.

The condition of compact support, unfamiliar from ordinary analysis, invites some explanation. For an instructive example [10], consider the noncompact supermanifold of functions: $] 0,1\left[\rightarrow \Lambda \mathbb{R}^{2}\right.$, with canonical coordinates $x, \xi^{1}, \xi^{2}$. If we choose to integrate with the Berezin form $\mathrm{d} x \partial^{2} / \partial \xi^{2} \partial \xi^{1}$, a superfunction $f$ that depends only on the combination $x+\xi^{1} \xi^{2}$ has the Berezin integral

$$
\int_{0}^{1} \mathrm{~d} x \frac{\partial^{2}}{\partial \xi^{2} \partial \xi^{1}} f\left(x+\xi^{1} \xi^{2}\right)=\int_{0}^{1} \mathrm{~d} x f^{\prime}(x)=f(1)-f(0) .
$$

On the other hand, if we made a change of integration variables

$$
y=x+\xi^{1} \xi^{2}, \quad \eta^{1}=\xi^{1}, \quad \eta^{2}=\xi^{2},
$$

which is easily seen to have unit Berezinian, we would get

$$
\int_{0}^{1} \mathrm{~d} x \frac{\partial^{2}}{\partial \xi^{2} \partial \xi^{1}} f\left(x+\xi^{1} \xi^{2}\right) \stackrel{? ?}{=} \int_{0}^{1} \mathrm{~d} y \frac{\partial^{2}}{\partial \eta^{2} \partial \eta^{1}} f(y)=0 .
$$

Because the Grassmann derivatives $\partial^{2} / \partial \eta^{2} \partial \eta^{1}$ have nothing to act on, the right hand side vanishes although for equality with the left hand side, it ought to be $f(1)-f(0)$. However, both sides vanish, and the discrepancy disappears, if we take $f$ to be compactly supported (which implies $f(0)=f(1)=0$ ), as is required in order for Berezin's theorem to apply. An alternative way of fixing the problem is to pass from the noncompact interval ]0, 1 [ to the compact circle $\mathrm{S}^{1}$, by imposing periodic boundary conditions on $x$, so that $f(1)-f(0)=0$.

The above example signals a general complication, which one has to confront when changing variables in a superintegral. While ordinary volume forms transform simply by the Jacobian, the transformation law for Berezin forms is not given only by the Berezinian, but involves an additional, "anomalous" term $\beta$ :

$$
D(y, \eta)=D(x, \xi) \operatorname{Ber}\left(\frac{y, \eta}{x, \xi}\right)+\beta,
$$


whenever some of the even coordinates are shifted by nilpotents. In our simple example,

$$
D(y, \eta) f=D(x, \xi) f+\left.\mathrm{d} x \frac{\partial}{\partial x} f\right|_{\xi^{1}=\xi^{2}=0} .
$$

The anomaly $\beta$ always has the property of being exact (in the sense of differential forms), i.e. $p[f]=\mathrm{d} \alpha[f]$ where $\mathrm{d} \alpha[f]$ is the exterior derivative of some $(p-1)$-form $\alpha[f]$. Therefore, by Stokes' theorem the anomaly can be integrated and converted into an integral over the boundary of the base of the supermanifold (or the boundary of the chart used, if there exist coordinate singularities). Rothstein [10 has given an explicit expression for the anomaly in terms of the vector field generating the transformation from $x, \xi$ to $y, \eta$.

\section{Supersymmetric formula for maps (II)}

After this brief tour of some basic supermathematics, we return to the task of setting up a supersymmetric formula for the pair correlations of quantized symplectic maps. We left off at Eq. (3) expressing $\Omega$ as a trace over $S$, the subspace of super Fock space $\mathcal{B}_{N}^{+} \otimes \mathcal{F}_{N}^{+} \otimes \mathcal{B}_{N}^{-} \otimes \mathcal{F}_{N}^{-}$determined by the condition $N_{+}=N_{-}$(equal number of retarded and advanced particles).

As was motivated at the example of the purely fermionic case in Sect. 4, our goal is now to trade the trace over $S$ for an integral over generalized coherent states. To that end, we observe that every Fock state $|\psi\rangle$ satisfying $\left(N_{+}-N_{-}\right)|\psi\rangle=0$ can be obtained by repeatedly acting on the vacuum with the pair creation operators $b_{+n}^{\dagger} b_{-n^{\prime}}^{\dagger}, b_{+n}^{\dagger} f_{-n^{\prime}}^{\dagger}, f_{+n}^{\dagger} b_{-n^{\prime}}^{\dagger}$, and $f_{+n}^{\dagger} f_{-n^{\prime}}^{\dagger}\left(n, n^{\prime}=\right.$ $1, \ldots, N)$. This leads us to consider coherent states of the pairing form

$$
|Z\rangle:=\exp \sum_{n n^{\prime}}\left(b_{+n}^{\dagger} Z_{n n^{\prime}}^{\mathrm{BB}} b_{-n^{\prime}}^{\dagger}+b_{+n}^{\dagger} Z_{n n^{\prime}}^{\mathrm{BF}} f_{-n^{\prime}}^{\dagger}+f_{+n}^{\dagger} Z_{n n^{\prime}}^{\mathrm{FB}} b_{-n^{\prime}}^{\dagger}+f_{+n}^{\dagger} Z_{n n^{\prime}}^{\mathrm{FF}} f_{-n^{\prime}}^{\dagger}\right)|0\rangle,
$$

where mathematical consistency requires taking complex numbers for the

matrix elements $Z_{n n^{\prime}}^{\mathrm{BB}}, Z_{n n^{\prime}}^{\mathrm{FF}}$ and Grassmann generators for $Z_{n n^{\prime}}^{\mathrm{BF}}, Z_{n n^{\prime}}^{\mathrm{FB}}$. It is convenient to assemble these into a supermatrix

$$
Z=\left(\begin{array}{cc}
Z^{\mathrm{BB}} & Z^{\mathrm{BF}} \\
Z^{\mathrm{FB}} & Z^{\mathrm{FF}}
\end{array}\right)
$$


Then a short hand notation for the pair coherent states is

$$
|Z\rangle=\exp \left(c_{+}^{\dagger} Z c_{-}^{\dagger}\right)|0\rangle
$$

where the creation operators $c_{ \pm}^{\dagger}$ stand for both $b_{ \pm}^{\dagger}$ and $f_{ \pm}^{\dagger}$. We will also need the dual of a pair coherent state

$$
\langle\tilde{Z}|:=\langle 0| \exp \left(c_{-} \sigma \tilde{Z} c_{+}\right), \quad \tilde{Z}=\left(\begin{array}{cc}
\tilde{Z}^{\mathrm{BB}} & \tilde{Z}^{\mathrm{BF}} \\
\tilde{Z}^{\mathrm{FB}} & \tilde{Z}^{\mathrm{FF}}
\end{array}\right), \quad \sigma=\left(\begin{array}{cc}
1_{N} & 0 \\
0 & -1_{N}
\end{array}\right) .
$$

In [11] it was shown that, with a suitable choice of Berezin form $D(Z, \tilde{Z})$, the pair coherent states provide a resolution of the projector $P$,

$$
P=\int_{0}^{2 \pi} \frac{d \theta}{2 \pi} \exp i \theta\left(N_{+}-N_{-}\right)=\int D(Z, \tilde{Z})\langle\tilde{Z} \mid Z\rangle^{-1}|Z\rangle\langle\tilde{Z}|,
$$

where the domain of integration is fixed by the conditions

$$
\tilde{Z}^{\mathrm{FF}}=-Z^{\mathrm{FF}^{\dagger}}, \quad \tilde{Z}^{\mathrm{BB}}=+Z^{\mathrm{BB}^{\dagger}}, \quad \text { and } \quad 1-Z^{\mathrm{BB}^{\dagger}} Z^{\mathrm{BB}}>0 .
$$

This resolution follows from Schur's lemma, once it has been

demonstrated that the right hand side commutes with all bilinears in Fock operators that commute with the projector $P$. The crucial relation needed for the latter statement is the invariance of the Berezin integral under a left translation through $g=\left(\begin{array}{cc}A & B \\ C & D\end{array}\right) \in \mathrm{GL}(2 N \mid 2 N)$,

$$
\begin{aligned}
& \int D(Z, \tilde{Z}) f(Z, \tilde{Z})= \\
& \quad \int D(Z, \tilde{Z}) f\left((A Z+B)(C Z+D)^{-1},(C+D \tilde{Z})(A+B \tilde{Z})^{-1}\right)
\end{aligned}
$$

which determines the Berezin form $D(Z, \tilde{Z})$ to be the invariant superintegration measure on Efetov's model space II (or, using the terminology of [12], on a Riemannian symmetric superspace of type AIII/AIII). The invariant Berezin form $D(Z, \tilde{Z})$ turns out to be flat as a result of cancellations due to supersymmetry:

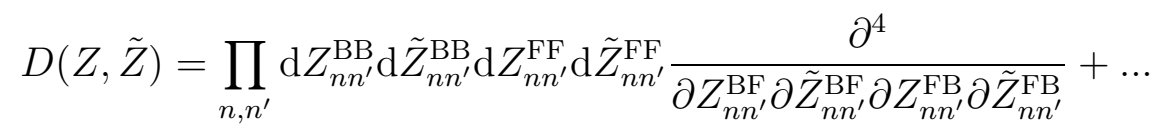


modulo boundary anomalies, which are indicated by the dots. The normalization of $D(Z, \tilde{Z})$ is fixed by $1=\langle 0|P| 0\rangle=\int D(Z, \tilde{Z})\langle\tilde{Z} \mid Z\rangle^{-1}$.

Given the coherent state resolution of the projector $P$, the trace of any operator $O$ on Fock space can be converted into a Berezin integral:

$$
\operatorname{Tr}_{N_{+}=N_{-}}(-1)^{N_{F}} O=\mathrm{S} \operatorname{Tr} P O=\int D(Z, \tilde{Z})\langle\tilde{Z}|O| Z\rangle /\langle\tilde{Z} \mid Z\rangle .
$$

Application to Eq. (3), followed by a computation of $\langle\tilde{Z} \mid Z\rangle$ and $\langle\tilde{Z}|\ldots| Z\rangle$, results in the desired formula for $\Omega$ :

$\Omega\left(\gamma_{+} ; \gamma_{-}\right)=\int D(Z, \tilde{Z}) \operatorname{SDet}(1-\tilde{Z} Z) \operatorname{SDet}^{-1}\left(1-\tilde{Z}\left(\gamma_{+} \otimes U_{N}\right) Z\left(\gamma_{-} \otimes U_{N}^{-1}\right)\right)$.

Here $\gamma_{ \pm}=\operatorname{diag}\left(\gamma_{ \pm 0}, \gamma_{ \pm 1}\right)$ are diagonal $2 \times 2$ matrices, and $\gamma_{+} \otimes U_{N}$ is meant to be a supermatrix:

$$
\gamma_{+} \otimes U_{N}=\left(\begin{array}{cc}
\gamma_{+0} U_{N} & 0 \\
0 & \gamma_{+1} U_{N}
\end{array}\right) .
$$

A closely related derivation of the formula for $\Omega$ is provided by the socalled color-flavor transformation ([11] and [13]). The two-level correlation function $R_{2}$ follows from $\Omega$ by taking two derivatives, as before.

\section{$7 \quad$ Semiclassical limit?}

So far, we have achieved no more (and no less) than an exact reformulation of the original problem. As should be clear from its derivation, the supersymmetric formula for the correlator $\Omega$ applies to any unitary matrix $U_{N}$, independently of whether this matrix arises from quantization of a map or not. In order to go further and extract nonempty information from our formalism, we need to exploit the fact that $U_{N}$ has a semiclassical limit and, in particular, we will have to make a distinction between chaotic and integrable systems.

To prepare these steps, we write $\Omega=\int D(Z, \tilde{Z}) e^{-S}$ where, borrowing the terminology from quantum field theory, the function

$$
S=-\mathrm{S} \operatorname{Tr} \ln (1-\tilde{Z} Z)+\mathrm{S} \operatorname{Tr} \ln \left(1-\tilde{Z}\left(\gamma_{+} \otimes U_{N}\right) Z\left(\gamma_{-} \otimes U_{N}^{-1}\right)\right)
$$

will be called the "action functional". Now recall that the BB- and FFblocks of $Z$ are complex $N \times N$ matrices. Viewing them as linear operators 
on the Hilbert space $\mathcal{H}_{N}$, we might say that they are similar to the density matrix of a quantum mechanical state. And, in fact, an expression like $Z^{\mathrm{BB}^{\prime}}:=U_{N} Z^{\mathrm{BB}} U_{N}^{-1}$, which occurs under the second logarithm in $S$, can be interpreted as being the "density matrix" $Z^{\mathrm{BB}}$ evolved by one (inverse) time step. One may therefore be tempted [4] to transform to a Wigner representation, postulate a semiclassical limit for $Z$ and subject the action functional to a semiclassical expansion. However, such an expansion is entirely uncontrolled in the present context and is, in fact, false. Unfortunately, a lot of confusion has been created among the community (including myself) concerning this point. Let me therefore change the style of presentation and give a detailed exposition of the issues as I see them. The reader is warned that, given the current level of understanding, the following has to be somewhat qualitative.

If $A$ is a linear operator (on some quantum mechanical Hilbert space) with kernel $A\left(q, q^{\prime}\right)$ in, say, the position representation, one defines the Wigner transform (or Weyl symbol) of $A$ by

$$
\sigma_{A}(q, p)=\sum_{q^{\prime}} A\left(q+q^{\prime} / 2, q-q^{\prime} / 2\right) e^{i p q^{\prime} / \hbar}
$$

whenever the transform exists. (In our case there is a technical complication due to the fact that the "position" on a compact phase space is not globally defined, but this is a peripheral issue and we are not going to worry about it here.) Now if $A$ and $B$ are pseudodifferential operators with Wigner transforms $\sigma_{A}(q, p)$ and $\sigma_{B}(q, p)$, their operator product has a semiclassical expansion

$$
\sigma_{A B}=\sigma_{A} \sigma_{B}+(\hbar / 2 i)\left\{\sigma_{A}, \sigma_{B}\right\}+\mathcal{O}\left(\hbar^{2}\right)
$$

where $\sigma_{A} \sigma_{B}$ is a product of functions, and the curly brackets denote the Poisson bracket.

The question now is whether this kind of expansion applies to products of the supermatrix $Z$. To be sure, there is nothing that prevents us from transforming $Z$ to a Wigner-like representation, but are we really allowed to make a semiclassical expansion of the action functional?! The question is a valid one, for the semiclassical expansion (6) does require $A$ and $B$ to be pseudodifferential operators. Without the smoothness property provided by this condition, the Wigner transform of the operator product $A B$ does not separate into the product of Wigner transforms $\sigma_{A} \sigma_{B}$ in the limit $\hbar \rightarrow 0$. For the standard operators appearing in quantum mechanics (position, momentum, 
energy etc.) the smoothness condition is of course always satisfied. However, there is no principle that guarantees smoothness of the Wigner transform $\sigma_{Z}$ in the present case. Indeed, $Z$ is not a fixed observable but is a variable of integration. While a small fraction of the $Z$ that are integrated over do correspond to smooth $\sigma_{Z}$, the vast majority do not. (Recall that the integral is over all complex supermatrices $Z, \tilde{Z}$ with the integration measure being that of a Riemanian symmetric superspace.) This invalidates the error estimate in (6). One may still cherish the hope that the dominant contributions to the $Z$-integral do come from smooth configurations. Admittedly, this is what happens for Feynman's path integral in the limit $\hbar \rightarrow 0$, which is dominated by paths that are extrema of the action functional. However, the present situation is quite different. There exists no saddle-point or stationary-phase or other principle here that would favor smoothness in general.

To recognize the severity of the problem, recall that the unitary operator $U_{N}$ has eigenvalues $e^{i \theta_{n}}$ and eigenfunctions $\psi_{n}$, and there exist $N$ of them. As a result, the conjugation $\operatorname{Ad}\left(U_{N}\right): \rho \mapsto U_{N} \rho U_{N}^{-1}$ has $N$ eigendensities $\rho=\psi_{n} \otimes \bar{\psi}_{n}$ with eigenvalues $e^{i\left(\theta_{n}-\theta_{n}\right)}=1$. To compute the pair correlation function in the microlocal scaling limit, we need to set $\gamma_{ \pm}=e^{i c_{ \pm}} / N$ and let $N$ go to infinity. In this limit the action functional acquires $N$ zero modes given by $Z=\sum_{n=1}^{N} C^{(n)} \psi_{n} \bar{\psi}_{n}$, where the $C^{(n)}$ are $2 \times 2$ supermatrices and are otherwise arbitrary. Thus there exist $N$ directions through the point $Z=\tilde{Z}=0$ along which the integrand is exactly neutral - a circumstance that surely causes very-large-amplitude fluctuations about this point. Moreover, the zero mode densities $\psi_{n} \bar{\psi}_{n}$ are never smooth. Indeed, in the integrable limit they are sharply localized on invariant sets, while in the chaotic limit they are highly irregular functions that vary on the shortest scales permitted by the uncertainty principle. In other words, the action functional (5) is unstable with respect to a large number $N$ of zero modes and none of these has a smooth Wigner transform. In view of all this, we had better abandon the hope that the dominant contributions to the $Z$-integral come from configurations that possess the smoothness of a pseudodifferential operator. Quite on the contrary, the most important configurations are those where $\sigma_{Z}$ varies with the shortest wave length possible! As $\hbar$ is lowered, the dominance of the $N$ zero modes keeps introducing an increasing number of relevant field configurations that vary on finer and finer scales. In order for the semiclassical asymptotics (6) to set in, we would have to intervene and limit the variations of $\sigma_{Z}$ to wave lengths that are greater than some fixed (i.e. $\hbar$-independent) minimal scale, and then let $\hbar$ go to zero. Without such an intervention, the 
Poisson bracket (and higher) terms in (6) fail to be $\hbar$-independent but diverge (with increasing powers) as $\hbar \rightarrow 0$, so that truncation of the semiclassical expansion of $S$ is totally unjustified. Consequently, any conclusion resulting from the use of this expansion in (5) is at risk to be false.

Given the invalidity of the semiclassical step, how can we use the supersymmetric formula (4) to develop the theory further? Not surprisingly, the answer depends on what we are trying to achieve. Here we are pursuing no more than the modest goal (modest from a physicist's perspective) of formulating a precise version of the BGS conjecture and substantiating it. As will be shown in the sequel, this restricted goal offers us the option of postponing the semiclassical step until the very end of the calculation.

\section{Regularization}

Notwithstanding the fact that the formula (4) is well-defined and, in fact, mathematically rigorous, we are facing a key difficulty: the existence of $N$ one-parameter groups along which the integrand lacks stability prevents us from investing semiclassical input and makes it difficult if not impossible to compute something from (4) as it stands. To make any progress at all, we must first solve this stability problem. Consider therefore, in the microlocal scaling limit $\gamma_{ \pm} \rightarrow 1$, the quadratic part of the action functional at $Z=\tilde{Z}=$ 0 :

$$
S^{(2)}=\mathrm{S} \operatorname{Tr} \tilde{Z} Z-\mathrm{S} \operatorname{Tr} \tilde{Z} U_{N} Z U_{N}^{-1}=\mathrm{S} \operatorname{Tr} \tilde{Z}\left(1-\operatorname{Ad}\left(U_{N}\right)\right) Z,
$$

which identifies the Hessian at this point as the operator $1-\operatorname{Ad}\left(U_{N}\right)$. Now recall that the classical limit of $\operatorname{Ad}\left(U_{N}\right)$ is $\chi^{*}$, the Frobenius-Perron operator of the inverse map $\chi^{-1}$. To prepare the discussion of the quantum mechanical operator $\operatorname{Ad}\left(U_{N}\right)$ in the limit $N \rightarrow \infty$, we shall summarize what we know about $\chi^{*}$.

First of all, because $\chi^{*}$ is unitary with respect to the Liouville $L^{2}$-measure on phase space, the spectrum of $\chi^{*}$ lies on the unit circle in $\mathbb{C}$. (If the map is mixing, the spectrum is known to be absolutely continuous [6].) Second, a salient feature of this spectrum is its instability with respect to regularization by smoothing. More precisely, if we project $\chi^{*}$ from the full $L^{2}$-space to a subspace of smooth functions, by coarse graining the phase space or imposing an ultraviolet (UV) cutoff, unitarity is lost and the spectrum moves to the interior of the unit circle. Third, if the map $\chi$ is mixing, the spectrum of the projected operator consists of one isolated nondegenerate eigenvalue at +1 
(with the corresponding eigenfunction being the invariant density $\rho_{0}=$ const) and so-called Ruelle resonances [14] inside the unit circle. It is important that this feature persists, i.e. the unitary spectrum is not recovered, when the UV cutoff is lowered to zero. (This is a statement of non-interchangability of limits.) Note also the difference between the quantum and classical cases: the quantum operator $1-\operatorname{Ad}\left(U_{N}\right)$ always has $N$ zero modes, whereas its classical limit $1-\chi^{*}$ on smooth functions only has a single zero mode for a mixing system, namely the invariant density.

The usual approach taken in the study of Ruelle resonances is to regularize in the ultraviolet by imposing a smoothness or differentiability condition on the phase space functions acted upon by the Frobenius-Perron operator. A similar effect is achieved if we leave the functions unchanged and, instead, implement the UV regularization on the Frobenius-Perron operator itself. For our purposes the latter procedure is more convenient. What we do is to make the replacement

$$
\chi^{*} \longrightarrow r^{\epsilon} \chi^{*},
$$

where the operator $r^{\epsilon}$ is chosen to be the exponential of a sum of squares of Hamiltonian vector fields $\Xi_{k}$,

$$
r^{\epsilon}=\exp \epsilon \sum_{k=1}^{s} \Xi_{k}^{2} .
$$

For example, for $M=\mathrm{S}^{2}$ we take for $\Xi_{k}$ the generators of rotations $(k=$ $1,2,3)$, in which case $\sum_{k=1}^{3} \Xi_{k}^{2}$ is simply the Laplacian on $\mathrm{S}^{2}$. More generally, the choice of Hamiltonian vector fields is constrained by the requirement that $\sum_{k} \Xi_{k}^{2}$ be an elliptic operator. The effect of $r^{\epsilon}$, then, is to regularize in the ultraviolet by suppressing fluctuations on short scales. It is important that $r^{\epsilon}$ is the exponential of a differential operator of second order, whereas the Frobenius-Perron operator can be thought of as the exponential of a firstorder differential operator. Therefore, $r^{\epsilon}$ is always relevant in the ultraviolet, no matter how small is the value of $\epsilon$. As a result, $r^{\epsilon}$ remains effective as a regulator even in the limit $\epsilon \rightarrow 0$ and, again, the unitary spectrum is not retrieved when the UV cutoff is removed.

In the following we will make use of the fact that, if the map $\chi$ is mixing, the operator $1-r^{\epsilon} \chi^{*}$ is strictly positive on the orthogonal complement of the uniform state $\rho_{0}$. The physical reason for such behavior is this. Consider the long time dynamics obtained by iterating $r^{\epsilon} \chi^{*}$ and, for simplicity of the argument, assume $\chi$ to be uniformly hyperbolic, which means that $\chi$ is 
everywhere stretching along unstable manifolds and contracting along stable ones. Thanks to the ellipticity of $\sum_{k=1}^{r} \Xi_{k}^{2}$, the operator $r^{\epsilon}$ counteracts the contraction along the stable manifolds via diffusion. After many iterations, the combination of stretching by $\chi$ and smoothing by $r^{\epsilon}$ will attract any initial state to the uniform state. Thus, as time $l$ goes to infinity, the operator $\left(r^{\epsilon} \chi^{*}\right)^{l}$ approaches the projector onto the uniform state $\rho_{0}$. This implies strict positivity of the real part of the spectrum of $1-r^{\epsilon} \chi^{*}$ operating on the orthogonal complement of $\rho_{0}$.

Let us now turn from the space of functions on $M$, acted upon by $\chi^{*}$, to the quantum mechanical space $\mathcal{H}_{N} \otimes \mathcal{H}_{N}^{*}$, acted upon by the operator $\operatorname{Ad}\left(U_{N}\right)$. Crudely speaking, the quantum space differs from the classical one by a finite resolution, or cutoff, set by Planck's constant. When this cutoff is much smaller than the regularization scale set by $\epsilon$, we have a good semiclassical expansion, (6), and the regularized $\operatorname{Ad}\left(U_{N}\right)$ spectrum looks qualitatively similar to the regularized Frobenius-Perron spectrum. On the other hand, if $\hbar$ is kept fixed at a finite value, the destruction of the unitary spectrum of $\operatorname{Ad}\left(U_{N}\right)$ with increasing $\epsilon$ is not abrupt but is rounded off. If we take $\epsilon \sim \hbar^{\alpha}$ with $\alpha>2 d$, the strength of the perturbation caused by the regulator becomes smaller than the level spacing of $\operatorname{Ad}\left(U_{N}\right)$ [which is $2 \pi / N^{2} \sim \hbar^{2 d}$, and is therefore negligible, in the limit $\hbar \rightarrow 0$. In contrast, if we choose the scaling exponent $\alpha$ in the range $0<\alpha<1$, then by power counting the regulator remains relevant for $\hbar \rightarrow 0$. This is good news: it suggests that we can kill the unitary spectrum of $\operatorname{Ad}\left(U_{N}\right)$, and thereby get rid of the zero mode problem if the classical map is mixing, by a regulator with strength $\epsilon$ that vanishes in the classical limit.

The basic strategy to follow should now be clear: our aim must be to substitute for the unitary operator $\operatorname{Ad}\left(U_{N}\right)$ in the quadratic functional $S^{(2)}$ the regularized operator $R_{N}^{\epsilon} \operatorname{Ad}\left(U_{N}\right)$, where $R_{N}^{\epsilon}$ is a quantization of the diffusion operator $r^{\epsilon}$. Such a substitution is expected to turn the unitary spectrum of $\operatorname{Ad}\left(U_{N}\right)$ into something akin to the Frobenius-Perron spectrum of the classical operator $\chi^{*}$. If $\chi$ is mixing and $\epsilon$ depends on $\hbar$ as $\epsilon \sim \hbar^{\alpha}$ with $0<\alpha<1$, only the invariant zero mode of $1-\operatorname{Ad}\left(U_{N}\right)$ remains neutral. The remaining $N-1$ zero modes turn into positive directions of the regularized Hessian and therefore become amenable to perturbative treatment. From experience with the application of the supersymmetric method to disordered electron systems [5], we then anticipate that a careful integration over the invariant zero mode will give the random matrix answer for the correlation functions.

What is unsatisfactory about this strategy is the absence of any explana- 
tion of where the $U V$ regularization came from. So far, regularization seems to be an ad hoc procedure, or "dirty trick", introduced so as to yield the result one wants to get. The situation would be different if we were working in a quantum field theoretic context. In that case, UV regularization would be well justified by the fundamental and inevitable existence of ultraviolet singularities (signaling incompleteness of the field theory, or "new physics" at short scales), which must be tamed by the introduction of a cutoff. In contrast, our formula (14) has no singularities whatsoever but is perfectly well-defined - we just don't know how to evaluate it - and there is no a priori need for regularization. In passing we mention that, if we ignored the arguments of Sect. 7 forbidding us to apply the semiclassical expansion (6) to the action functional $S$, and applied the expansion anyway, then we would, in fact, end up with an ultraviolet singular field theory [4]. However, the UV divergencies so introduced are entirely artificial - they simply confirm the fact that we have made improper use of the semiclassical expansion - and do not justify the postulate of any regulator.

To reiterate, it is illegal just to postulate UV regularization as a technical device or trick. For a convincing argument, one needs to explain exactly what modification of the original problem is implied by regularization, and one has to do that in a frame that precedes the supersymmetric quantum field theory formalism. As will be argued in the remainder of this section, regularization of the Hessian of (5) at $Z=\tilde{Z}=0$ amounts to averaging over the "quantum" ensemble described in Sect. 3.

Recall that, if $\xi_{k}(k=1, \ldots, s)$ are uncorrelated Gaussian random variables with zero mean and variance $\left\langle\xi_{k}^{2}\right\rangle=2 \epsilon$, the ensemble is specified by

$$
U_{N}(\xi)=\exp \left(i \sum_{k=1}^{s} \xi_{k} X_{k, N} / \hbar\right) U_{N}
$$

By averaging the Hessian $1-\operatorname{Ad}\left(U_{N}(\xi)\right)$ over the probability space of the parameters $\xi_{k}$, we get

$$
\left\langle 1-\operatorname{Ad}\left(U_{N}(\xi)\right)\right\rangle=1-R_{N}^{\epsilon} \operatorname{Ad}\left(U_{N}\right),
$$

where the operator $R_{N}^{\epsilon}$ is identified as

$$
\begin{aligned}
R_{N}^{\epsilon} & =\left\langle\operatorname{Ad}\left(\exp i \sum_{k=1}^{s} \xi_{k} X_{k, N} / \hbar\right)\right\rangle \\
& =\left\langle\exp i \sum_{k} \xi_{k} \operatorname{ad}\left(X_{k, N}\right) / \hbar\right\rangle=\exp -\epsilon \sum_{k} \operatorname{ad}^{2}\left(X_{k, N}\right) / \hbar^{2}+\ldots
\end{aligned}
$$


[Note that, since the integrand in (ब1) depends on $U_{N}$ in a nonlinear way, later we will have to confront averages of powers of $\operatorname{Ad}\left(U_{N}(\xi)\right)$. For the time being, we concentrate on the Hessian.] The terms indicated by dots in the last expression become negligible in the semiclassical limit $\hbar \rightarrow 0$. Taking the commutator with $X_{k, N}$ in this limit corresponds to applying the Hamiltonian vector field $\Xi_{k}$ :

$$
\operatorname{ad}\left(X_{k, N}\right)=\left[X_{k, N}, \bullet \stackrel{\hbar \rightarrow 0}{\longrightarrow} i \hbar \Xi_{k},\right.
$$

so $R_{N}^{\epsilon}$ is in fact a quantization of the classical diffusion operator $e^{\epsilon \sum_{k=1}^{s} \Xi_{k}^{2}}=$ $r^{\epsilon}$, as desired.

To conclude, averaging over the specified ensemble regularizes the Hessian in the ultraviolet. As was argued earlier, if the classical map $\chi$ is mixing the real part of $1-r^{\epsilon} \chi^{*}$ is positive on all states that are orthogonal to the uniform state $\rho_{0}$. In view of the semiclassical limits $R_{N}^{\epsilon} \rightarrow r^{\epsilon}$ and $\operatorname{Ad}\left(U_{N}\right) \rightarrow \chi^{*}$, we expect the same to be true for the regularized Hessian $1-R_{N}^{\epsilon} \operatorname{Ad}\left(U_{N}\right)$ on $\mathcal{D}_{N}^{\perp}$, the subspace of $\mathcal{H}_{N} \otimes \mathcal{H}_{N}^{*}$ orthogonal to the invariant element. This key observation allows us to proceed as follows.

\section{$9 \quad$ Asymptotic expansion}

We set $\gamma_{ \pm}=e^{ \pm i c_{ \pm} / N}$ where $c_{ \pm}=\operatorname{diag}\left(c_{ \pm 0}, c_{ \pm 1}\right)$ are $2 \times 2$ matrices and, to abbreviate the notation, we put $Z_{U}:=\left(1_{2} \otimes U_{N}\right) Z\left(1_{2} \otimes U_{N}^{-1}\right)$. Then, since $c_{ \pm}$ are held fixed while $N$ is sent to infinity, we may expand the action functional:

$$
\begin{aligned}
S= & -\mathrm{S} \operatorname{Tr} \ln (1-\tilde{Z} Z)+\mathrm{S} \operatorname{Tr} \ln \left(1-\tilde{Z} Z_{U}\right) \\
& -\frac{i}{N} \mathrm{~S} \operatorname{Tr}\left(c_{+} Z_{U} \tilde{Z}\left(1-Z_{U} \tilde{Z}\right)^{-1}-c_{-} \tilde{Z} Z_{U}\left(1-\tilde{Z} Z_{U}\right)^{-1}\right)+\mathcal{O}\left(1 / N^{2}\right) .
\end{aligned}
$$

To isolate the invariant zero mode, we make the substitution

$$
\begin{aligned}
& Z=\left(\zeta+Z_{0}\right)\left(1+\tilde{Z}_{0} \zeta\right)^{-1} \\
& \tilde{Z}=\left(\tilde{\zeta}+\tilde{Z}_{0}\right)\left(1+Z_{0} \tilde{\zeta}\right)^{-1}
\end{aligned}
$$

The supermatrices $\zeta$ and $\tilde{\zeta}$ are taken to be traceless in each block (i.e. $\operatorname{Tr} \zeta^{\sigma \tau}=\operatorname{Tr} \tilde{\zeta}^{\sigma \tau}=0$ for $\sigma, \tau=\mathrm{B}, \mathrm{F}$ ), and the zero mode is parameterized by $Z_{0}=z_{0} \otimes 1_{N}, \tilde{Z}_{0}=\tilde{z}_{0} \otimes 1_{N}$ with $2 \times 2$ matrices

$$
z_{0}=\left(\begin{array}{ll}
z_{0}^{\mathrm{BB}} & z_{0}^{\mathrm{BF}} \\
z_{0}^{\mathrm{FB}} & z_{0}^{\mathrm{FF}}
\end{array}\right), \quad \tilde{z}_{0}=\left(\begin{array}{cc}
\tilde{z}_{0}^{\mathrm{BB}} & \tilde{z}_{0}^{\mathrm{BF}} \\
\tilde{z}_{0}^{\mathrm{FB}} & \tilde{z}_{0}^{\mathrm{FF}}
\end{array}\right) .
$$


Because the transformation

$$
\begin{aligned}
Z & \mapsto(A Z+B)(C Z+D)^{-1} \\
\tilde{Z} & \mapsto(C+D \tilde{Z})(A+B \tilde{Z})^{-1}
\end{aligned}
$$

is an isometry of the Riemannian symmetric superspace, the Berezinian of the change of variables from $Z$ to $\left(\zeta, Z_{0}\right)$ is unity and $D(Z, \tilde{Z})$ factors into a product of Berezin forms $D\left(z_{0}, \tilde{z}_{0}\right) D(\zeta, \tilde{\zeta})$. (We pay no attention to possible boundary anomalies here.) Now, by inserting the expansion of the action $S$ into (4), changing variables as indicated, and using identities such as

$$
\left(1-Z_{U} \tilde{Z}\right)^{-1}=\left(1+Z_{0} \tilde{\zeta}\right)\left(1-\zeta_{U} \tilde{\zeta}\right)^{-1}\left(1+\zeta_{U} \tilde{Z}_{0}\right)\left(1-Z_{0} \tilde{Z}_{0}\right)^{-1}
$$

we obtain

$$
\begin{aligned}
\Omega\left(e^{i c_{+} / N} ; e^{-i c_{-} / N}\right) & =\int D\left(z_{0}, \tilde{z}_{0}\right) \Omega_{1}\left(c_{+} / N, c_{-} / N ; z_{0}, \tilde{z}_{0}\right) \\
& \times \exp i \operatorname{STr}_{\mathbb{C}^{1 \mid 1}}\left(c_{+} z_{0} \tilde{z}_{0}\left(1-z_{0} \tilde{z}_{0}\right)^{-1}-c_{-} \tilde{z}_{0} z_{0}\left(1-\tilde{z}_{0} z_{0}\right)^{-1}\right)
\end{aligned}
$$

with $\Omega_{1}$ defined by

$$
\begin{aligned}
& \Omega_{1}\left(c_{+} / N, c_{-} / N ; z_{0}, \tilde{z}_{0}\right) \\
= & \int D(\zeta, \tilde{\zeta}) \operatorname{SDet}(1-\tilde{\zeta} \zeta) \operatorname{SDet}^{-1}\left(1-\tilde{\zeta} \zeta_{U}\right) \\
& \times \exp \left(\frac{i}{N} \operatorname{STr} c_{+}\left(1+Z_{0} \tilde{\zeta}\right)\left(1-\zeta_{U} \tilde{\zeta}\right)^{-1} \zeta_{U}\left(\tilde{\zeta}+\tilde{Z}_{0}\right)\left(1-Z_{0} \tilde{Z}_{0}\right)^{-1}\right. \\
& \left.-\frac{i}{N} \operatorname{STr} c_{-}\left(1+\tilde{Z}_{0} \zeta_{U}\right)\left(1-\tilde{\zeta} \zeta_{U}\right)^{-1} \tilde{\zeta}\left(\zeta_{U}+Z_{0}\right)\left(1-\tilde{Z}_{0} Z_{0}\right)^{-1}\right) .
\end{aligned}
$$

Note that convergence of the last integral is ensured by the condition $\operatorname{Im} c_{+0}>$ $0>\operatorname{Im} c_{-0}$ resulting from $\left|\gamma_{ \pm 0}\right|<1$.

Our next goal is to establish an asymptotic $1 / N$ expansion for the function $\Omega_{1}$. To that end, we temporarily set $c_{+} / N=c_{-} / N=0$ and consider the integral

$$
\Omega_{1}\left(0,0 ; z_{0}, \tilde{z}_{0}\right)=\int D(\zeta, \tilde{\zeta}) \operatorname{SDet}(1-\tilde{\zeta} \zeta) \operatorname{SDet}^{-1}\left(1-\tilde{\zeta} \zeta_{U}\right)
$$

To compute this, we observe that both the Berezin form $D(\zeta, \tilde{\zeta})$ and the two superdeterminants are invariant under transformations

$$
\zeta \mapsto A \zeta D^{-1}, \quad \tilde{\zeta} \mapsto D \tilde{\zeta} A^{-1}
$$


with $A=a \otimes 1_{N}$ and $D=d \otimes 1_{N}$. (Indeed, such transformations commute with $\operatorname{Ad}\left(U_{N}\right)$ and are isometries of the symmetric superspace.) This invariance results in the value of the integral being equal to the value of the integrand at the origin $\zeta=\tilde{\zeta}=0$ :

$$
\Omega_{1}\left(0,0 ; z_{0}, \tilde{z}_{0}\right)=1,
$$

by what is often called the Parisi-Sourlas-Efetov-Wegner theorem in disordered electron physics. The mechanism underlying the theorem is called "localization" in the mathematics literature. In a setting closely related to equivariant cohomology, localization of superintegrals has recently been discussed by Schwartz and Zaboronsky [15. With reference to their results we can argue as follows. On setting

$$
A=\exp \left(\begin{array}{cc}
0 & \alpha \\
\bar{\alpha} & 0
\end{array}\right) \otimes 1_{N}, \quad D=\exp \left(\begin{array}{cc}
0 & \delta \\
\bar{\delta} & 0
\end{array}\right) \otimes 1_{N}
$$

and differentiating with respect to the Grassmann parameters $\alpha, \bar{\alpha}, \delta, \bar{\delta}$, the transformation $\zeta \mapsto A \zeta D^{-1}, \tilde{\zeta} \mapsto D \tilde{\zeta} A^{-1}$ gives rise to four odd vector fields, each of which leaves the integrand invariant. The joint zero locus of these vector fields is $\zeta=\bar{\zeta}=0$, and the superdeterminant of the Hessian at this point is unity by cancellation due to supersymmetry. Moreover, the vector fields are compact in the sense of Schwartz and Zaboronsky. Therefore, by the version of the Parisi-Sourlas-Efetov-Wegner theorem proved in [15, the integral equals unity as claimed.

Given the result $\Omega_{1}\left(0,0 ; z_{0}, \tilde{z}_{0}\right)=1$, one would like to proceed and make a saddle point approximation around $\zeta=\tilde{\zeta}=0$, to produce an asymptotic expansion for $\Omega_{1}\left(c_{+} / N, c_{-} / N ; z_{0}, \tilde{z}_{0}\right)$ of the form $1+N^{-1} f\left(c_{+}, c_{-} ; z_{0}, \tilde{z}_{0}\right)+$ $\mathcal{O}\left(N^{-2}\right)$. However, this is impossible as it stands. The reason is the notorious problem of $N-1$ zero modes: there exists an $(N-1)$-dimensional maximal torus of group actions that commute with $\operatorname{Ad}\left(U_{N}\right)$ and leave $\operatorname{SDet}(1-$ $\tilde{\zeta} \zeta) \operatorname{SDet}^{-1}\left(1-\tilde{\zeta} \zeta_{U}\right)$ invariant, thereby causing a lack of stability of the candidate saddle point $\zeta=\tilde{\zeta}=0$. Fortunately, given all the preparations that were made in Sect. 8, we immediately know how to fix this stability problem.

We substitute $U_{N}(\xi)$ for $U_{N}$ and average over the probability space of the ensemble, replacing $\Omega$ by $\langle\Omega\rangle$, and $\Omega_{1}$ by $\left\langle\Omega_{1}\right\rangle$. The integral for $\Omega_{1}$ converges and, as a matter of fact, converges uniformly in the parameters $\xi_{k}$, if $\operatorname{Im} c_{+0} \geq 0 \geq \operatorname{Im} c_{-0}$. Therefore, we may interchange the order of integration and ensemble averaging. By the same localization argument as 
before,

$$
\left\langle\Omega_{1}\left(0,0 ; z_{0}, \tilde{z}_{0}\right)\right\rangle=\int D(\zeta, \tilde{\zeta})\left\langle\operatorname{SDet}(1-\tilde{\zeta} \zeta) \operatorname{SDet}^{-1}\left(1-\tilde{\zeta} \zeta_{U_{N}(\xi)}\right)\right\rangle=1 .
$$

What has improved in comparison with the situation without ensemble averaging is that now we do have a good asymptotic expansion. The reason is that integrals such as

$$
\begin{aligned}
& \int D(\zeta, \tilde{\zeta})\left\langle\operatorname{SDet}(1-\tilde{\zeta} \zeta) \operatorname{SDet}^{-1}\left(1-\tilde{\zeta} \zeta_{U_{N}(\xi)}\right)\right. \\
& \left.\times \operatorname{STr} c_{+}\left(1-\zeta_{U_{N}(\xi)} \tilde{\zeta}\right)^{-1} \zeta_{U_{N}(\xi)} \tilde{\zeta}\left(1-Z_{0} \tilde{Z}_{0}\right)^{-1}\right\rangle
\end{aligned}
$$

are expected to exist and remain finite in the limit $N \rightarrow \infty$. This can be checked by expanding around the saddle point $\zeta=\tilde{\zeta}=0$ in the usual manner. By doing the resulting Gaussian integrals over $\zeta, \tilde{\zeta}$, one obtains traces involving powers of the inverse of the regularized Hessian $1-R_{N}^{\epsilon} \operatorname{Ad}\left(U_{N}\right)$ acting on $\mathcal{D}_{N}^{\perp}$. We have argued earlier that the regularized Hessian remains strictly positive in the semiclassical limit if the classical map is mixing. Therefore, the inverse of $1-R_{N}^{\epsilon} \operatorname{Ad}\left(U_{N}\right)$ exists on $\mathcal{D}_{N}^{\perp}$ and is uniformly bounded in $N$. Moreover, as follows from semiclassical trace formulas, the traces encountered are bounded uniformly in $N$ if the fixed points of the classical map are isolated.

What all this amounts to is that, unlike $\Omega_{1}$, the ensemble average $\left\langle\Omega_{1}\right\rangle$ does have an asymptotic expansion:

$$
\left\langle\Omega_{1}\left(c_{+} / N, c_{-} / N ; z_{0}, \tilde{z}_{0}\right)\right\rangle=1+N^{-1} f\left(c_{+}, c_{-} ; z_{0}, \tilde{z}_{0}\right)+\mathcal{O}\left(N^{-2}\right) .
$$

Using this in the formula for the correlator $\langle\Omega\rangle$ we obtain

$$
\begin{aligned}
& \lim _{N \rightarrow \infty}\left\langle\Omega\left(e^{i c_{+} / N} ; e^{-i c_{-} / N}\right)\right\rangle \\
= & \int D\left(z_{0}, \tilde{z}_{0}\right) \exp i \operatorname{STr}\left(c_{+} z_{0} \tilde{z}_{0}\left(1-z_{0} \tilde{z}_{0}\right)^{-1}-c_{-} \tilde{z}_{0} z_{0}\left(1-\tilde{z}_{0} z_{0}\right)^{-1}\right) .
\end{aligned}
$$

Computation of this definite superintegral is a standard exercise and gives the random matrix answer (1). The result for the pair correlation function follows by taking two derivatives. The dependence on $z_{0}, \tilde{z}_{0}$ of the $1 / N$ correction to $\left\langle\Omega_{1}\right\rangle$ can be shown to be such that the integral over $z_{0}, \tilde{z}_{0}$ exists and the coefficient of $1 / N$ in $\langle\Omega\rangle$ is finite.

In conclusion, I believe that the arguments presented here are strong evidence in favor of the precise version of the BGS conjecture proposed. To turn them into a proof that is rigorous by mathematical standards, three improvements are required. 
1. One needs to do a spectral analysis of the operator $R_{N}^{\epsilon} \operatorname{Ad}\left(U_{N}\right)$ to support our intuition of what happens in the limit $N \rightarrow \infty$.

2. The transformation of Berezin forms $D(Z, \tilde{Z}) \rightarrow D\left(z_{0}, \tilde{z}_{0}\right) D(\zeta, \tilde{\zeta})$ needs to be scrutinized carefully, to rule out the possible existence of boundary anomalies (Sect. 5) that might interfere with our argument.

3. A complete computation of the $1 / N$ corrections is called for. In particular, one must resolve the puzzle why the calculation of Andreev et al, which is closely related to ours, fails to reproduce the diagonal approximation to the double sum over periodic orbits.

Acknowledgment. While participating in the workshop on "Disordered Systems and Quantum Chaos" at the Newton Institute, Cambridge, where these lectures were delivered, I enjoyed a number of illuminating discussions with Steve Zelditch, who got me straightened out on the subject of semiclassical limits and suggested to compose the deterministic map with a stochastic Hamiltonian flow that averages to a diffusion operator.

\section{References}

[1] O. Bohigas, M.J. Giannoni, and C. Schmit, Phys. Rev. Lett. 52, 1 (1984).

[2] M.V. Berry, Proc. R. Soc. London A 400, 229 (1985).

[3] E.B. Bogomolny and J.P. Keating, Phys. Rev. Lett. 77, 1472 (1996).

[4] A.V. Andreev, B.D. Simons, O. Agam, and B.L. Altshuler, Nucl. Phys. B 482, 536 (1996).

[5] K.B. Efetov, Adv. Phys. 32, 53 (1983).

[6] V.I. Arnold and A. Avez, Ergodic Problems of Classical Mechanics (Benjamin, New York, 1968).

[7] A.M. Perelomov, Generalized coherent states and their applications (Springer-Verlag, Berlin, 1986).

[8] F.A. Berezin and D.A. Leites, Sov. Math. Dokl. 16, 1218 (1975); B. Kostant, Lect. Notes Math. 570, 177 (1977). 
[9] F.A. Berezin, Introduction to Superanalysis (Reidel, Dordrecht, 1987).

[10] M.J. Rothstein, Trans. Amer. Math. Soc. 299, 387 (1987).

[11] M.R. Zirnbauer, J. Phys. A 29, 7113 (1996).

[12] M.R. Zirnbauer, J. Math. Phys. 37, 4986 (1996).

[13] M.R. Zirnbauer, ICMP97 proceedings; chao-dyn/9810016.

[14] D. Ruelle, Phys. Rev. Lett. 56, 405 (1988).

[15] A. Schwartz and O. Zaboronsky, Commun. Math. Phys. 183, 463 (1997). 